\title{
La figure du contrat dans la décentralisation de la gestion des ressources naturelles
}

Martine Antona, Laurence Boutinot et Brehima Kassibo

\section{(2) OpenEdition}

\section{Journals}

Édition électronique

URL : http://journals.openedition.org/anthropodev/474

DOI : 10.4000/anthropodev.474

ISSN : 2553-1719

Éditeur

APAD - Association pour l'anthropologie du changement social et du développement

Édition imprimée

Date de publication : 1 avril 2014

Pagination : 121-156

ISBN : 979-10-93476-00-1

ISSN : 2276-2019

Référence électronique

Martine Antona, Laurence Boutinot et Brehima Kassibo, " La figure du contrat dans la décentralisation de la gestion des ressources naturelles », Anthropologie \& développement [En ligne], 37-38-39| 2014, mis en ligne le 01 décembre 2016, consulté le 05 mai 2019. URL : http://journals.openedition.org/ anthropodev/474; DOI : 10.4000/anthropodev.474

La revue Anthropologie \& développement est mise à disposition selon les termes de la Licence Creative Commons Attribution 4.0 International. 


\title{
La figure du contrat dans la décentralisation de la gestion des ressources naturelles
}

\author{
Martine Antona, Laurence Boutinot, Brehima Kassibo
}

Sous la figure du contrat, nous classons les procédures d'élaboration des conventions locales qui visent à coordonner des acteurs aux intérêts divers en vue d'un objectif commun de gestion d'une ressource (forestière, halieutique) sur des espaces identifiés. Au Mali, au Niger et au Sénégal, ces conventions locales de gestion sont mises en cuvre dans le cadre de grands programmes de la Banque mondiale pour la gestion durable des forêts et la production de l'énergie domestique (bois, charbon de bois). Les théories néoinstitutionnelles inspirent aux bailleurs de fonds des contrats de gestion qui sont censés promouvoir les actions collectives et réduire les asymétries d'information, les comportements opportunistes et les coûts de transaction. Or, une approche croisée en économie et en socio-anthropologie donne à voir que ces contrats sont peu respectés, donnent parfois lieu à des conflits et sont appliqués à partir de normes techniques et standardisées, faisant fi des contextes sociaux et politiques locaux et nationaux. A l'échelle politique de la gouvernance locale, ces contrats multiplient les lieux de décision et d'autorité et viennent contrarier les procédures de délégation de compétences aux collectivités locales dans le cadre de la décentralisation.

Under the figure of the contract, are described the procedures for developing local decentralized policies that aim to coordinate actors with multiple interests towards a common goal of a resource management (forestry, fisheries) on defined areas. In Mali, Niger and Senegal, these local management agreements are implemented as part of World Bank major programs for sustainable forest management and domestic energy production (wood, charcoal). New institutional approaches inspired donors to design management policies as contracts between State bodies and local representatives of the end -users. The contracts are supposed to promote collective action and to reduce information asymmetries, opportunistic behavior 
and transaction costs. We crossed anthropological and economical insights on the low compliance with these contracts, which sometimes led to conflict, are implemented through technical and standardized norms, and without considering local and national social and political contexts. At the political level of local governance, these contracts increase decision-making places and upset the procedures for delegating powers to local governments as required in the context of decentralization.

\section{Introduction}

De nouvelles formes de gestion décentralisée des ressources naturelles sont mises en œuvre au Sud depuis le début des années 1990 (Ribot 2002, Larson 2006, Coleman 2001). Elles sont largement inspirées des agendas des institutions internationales et des bailleurs de fond, notamment à partir du constat de l'échec de la gestion centralisée des États africains dans de nombreux secteurs dont ceux des forêts et de la pêche (Crook et Manor 1998). Les bailleurs de fonds, les États et les acteurs privés ont promu la figure du contrat dans les contextes de décentralisation et de libéralisation économique. Celle-ci fait suite à l'encadrement du monde paysan, des premières structures coopératives au désengagement des États, et s'inscrit depuis vingt ans dans la logique des projets de développement " communautaires " et " participatifs ». Sous la figure du contrat, nous classons toutes les procédures et démarches d'élaboration de chartes, de codes, de conventions locales qui visent à coordonner des acteurs aux intérêts divers en vue d'un objectif supposé commun de gestion sectorielle d'une ressource (forestière, halieutique) et des espaces concernés. Conclu entre les populations locales, les exploitants, les administrations, voire les élus locaux, le contrat vise à construire une coordination des intérêts des différentes parties prenantes et à fixer, de manière formelle, les engagements réciproques entre ces parties. Les dispositifs contractuels veulent répondre à un double mouvement de changement social que nous observons en Afrique notamment : d'un côté la perte de légitimité des modes coutumiers sur la gestion des ressources et des territoires, de l'autre le désengagement de l'État dans la délivrance des services publics (Bardhan, 2002). 
Dans les travaux menés sur le thème du « Natural Resource Management » (NRM) (Thomson 2000, Agrawal et Ostrom 2001, Blaikie, 2006), l'espace local où les projets de développement mobilisent la participation des villageois pour susciter une action collective et formaliser celle-ci dans des institutions locales ad hoc (comité, structure rurale ou locale de gestion), s'appelle l'espace de la dévolution (Bene, 2009 : 1938). Cet espace théorique (la dévolution) se situe entre la décentralisation territoriale qui concerne le transfert des compétences de l'État aux collectivités locales élues (communes urbaines, communes et communautés rurales) et la déconcentration des services publics sectoriels (ici les Eaux et Forêts) dans les régions. On assiste ainsi à un double processus de décentralisation politique et de dévolution dont les rapports sont variables selon les contextes (Agrawal et Ribot 1999, Meinzen Dick et al 1999, Ribot 2002, Bene 2009). Ces auteurs mettent en évidence le fait que l'intérêt pour la dévolution a coïncidé avec l'emphase mise sur la participation et la démocratisation. Souvent qualifiées de "co-management " dans la littérature, ces nouvelles formes de gouvernance revendiquent un changement de paradigme en termes de " systèmes de coordination non-hiérarchiques " et de rapports horizontaux (Ostrom, 1986, 2005 ; Hufty, 2007).

La littérature présente de nombreuses analyses de ces processus dans leur diversité afin de clarifier les concepts (décentralisation/dévolution/ " co-management "), et d'en proposer des critères pour l'analyse. Ribot et Agrawal développent ainsi en 1999 un cadre basé sur le tryptique " acteurs, pouvoirs, redevabilité ". Meinzen Dik et al. (1999) mettent en avant la redistribution des droits de propriété et la capacité d'action collective que les usagers doivent exercer pour assurer les rôles délégués par l'État. Coleman et Fleishman (2001), Gibson et Lehoucq (2003), Edmunds et Wollenberg (2004) et Larson (2005) s'appuient, quant à eux, sur les expériences de gestion décentralisée des forêts pour analyser leurs impacts sur l'amélioration de la gouvernance et sur les bénéfices qui en sont issus pour les usagers. Dans des publications précédentes du Bulletin de I'APAD, ces processus ont été décrits sous l'angle des rapports sociaux entre agents de l'État et usagers, de leurs représentations et pratiques respectives et sur la délivrance des services publics (Blundo 2002, BakoArifari et Laurent 1998).

Dans cet article, notre démarche n'est pas d'évaluer les dispositifs mis en place dans plusieurs pays d'Afrique de l'Ouest, mais de montrer 
comment, dans leur élaboration et leur fonctionnement, ils s'éloignent du paradigme du "co-management ", qui leur est souvent associé. Nous faisons l'hypothèse que si ce co-management est peu appliqué, voire contourné et donne lieu à de nombreux conflits, on peut l'expliquer par une vision techniciste et standardisée de la gestion des forêts, mais aussi par des concepts d'inspiration individualiste et " dés enchâssés " des rapports sociaux.

Nous nous appuierons sur des enquêtes menées dans trois pays: le Mali, le Sénégal et le Niger. Ces pays se sont engagés dans ce double processus de décentralisation et de transfert contractuel de la gestion des forêts, à plus ou moins grande échelle. Les enquêtes ont été menées au moment de la mise en place des comités villageois au Sénégal (2004-2007), au Mali (2000-2001) et entre 1999 et 2004 au Niger, soit plus de 10 ans après le démarrage des dispositifs de marchés ruraux. Au Sénégal, pas moins d'une vingtaine de types de conventions étaient en application sur l'ensemble du territoire national en 2003 (Granier 2003), le Mali comptait 100 dispositifs de marchés ruraux en 2004 (300 en 2009) et au Niger 180 (400 en 2009). Le cas de chaque pays présente aussi l'intérêt d'offrir un gradient dans l'insertion des contrats dans les politiques publiques qui mixe les concepts de dévolution et de décentralisation (Noppen et al. 2004, Bertrand et Montagne 2009). Ces formes de contractualisation ont pour fonction à la fois de produire une certaine demande de sécurisation juridique sur l'accès aux ressources émanant des acteurs villageois, et d'y répondre (Granier 2003, Djiré 2004). Dans le même temps, elles font reposer sur ces mêmes acteurs, une partie des coûts de maintien, d'usage et d'entretien des ressources. Enfin, elles rencontrent également la volonté des services de l'État de maintenir leur contrôle sur les processus de transfert de pouvoir aux acteurs villageois et aux collectivités locales, au-delà des textes de lois (Ribot 1990, Kassibo 1997, Gautier et al. 2008, Bertrand et Montagne 2009, Hautdidier et al. 2010).

\section{Croiser des approches}

Nous mobilisons ici les analyses de l'économie et de l'anthropologie pour comprendre les tensions au centre des dispositifs contractuels. L'élaboration et le fonctionnement de ces contrats, qui supposent un partage de pouvoirs, posent les questions du " politique » dans le diagnostic 
de la situation initiale et partant, de la répartition des pouvoirs à l'issue des processus de contractualisation. Le contrat s'établit bien souvent à partir d'échanges d'informations sur les droits et ressources et sur la base d'une vision du contexte quelque peu abstraite, technique et exogène aux acteurs. Quand le contexte est considéré, il est analysé à l'aune d'un principe normatif de qualité du milieu (nombre d'arbres à l'hectare, composition par essences forestières, âge et diamètre, etc.) qui favoriserait sa durabilité, et partant, un compromis sur le futur ; ce que les cas étudiés amènent à interroger.

Dans les approches de l'économie néo-institutionnelle, l'élaboration des contrats renvoie d'une part, à une offre de décentralisation qui reformule les rapports entre l'État et les autres acteurs, tout en visant l'efficacité de l'action de l'État; et d'autre part, à un problème technique de " design " du contrat, de contrôle des co-contractants et de mesure de ses effets. Selon cette théorie, le comportement d'un acteur dépendrait de sa capacité à calculer et anticiper le comportement des autres. Le contrat est un arrangement institutionnel qui tente de résorber les comportements opportunistes, de réduire et les asymétries d'information et le coût du suivi et du contrôle des actions (Coase, 1937). Ce qui est recherché dans le contrat est alors un système d'incitation susceptible de rendre compatibles les intérêts des co-contractants. Appliqué à l'échelle de la dévolution, le contrat est censé répondre aux problèmes d'information et d'incitations négatives par une répartition des capacités (responsabilités) et des ressources entre les parties prenantes y compris le régulateur (ici l'administration forestière) et les acteurs bénéficiaires du transfert d'autorité sur les forêts (Birner et al. 2004, Anderson et al. 2006).

L'économie institutionnelle, proche de l'économie du développement s'écarte, quant à elle, de cette conception calculatrice de la coordination entre acteurs. Le présupposé selon lequel les institutions sont créées pour économiser des coûts de transaction et sont efficientes, est battu en brèche. La dynamique institutionnelle relèverait plus d'un déséquilibre dans les pouvoirs et d'une émergence de nouvelles valeurs sociales (North 1990). Les arrangements institutionnels dans différents contextes sont analysés à partir des capacités locales (le " capital social »), des normes de comportements individuels (confiance, réciprocité) qui régissent les interactions entre des acteurs (nestedness) de différents niveaux de gouvernance (Ostrom 2005, Ostrom 2010). Dans tous les cas, ces diverses 
approches, centrées sur la réaction des acteurs face à des incitations, apportent des réponses limitées et abstraites à la question de la procédure de négociation, censée aboutir à la coordination des intérêts, qui est au cœur de la contractualisation.

Les conventions locales de gestion, les contrats ou les chartes ne sont des objets d'étude pour l'anthropologue qu'en tant qu'ils rendent compte des rapports sociaux. C'est le cas ici du champ de la gestion des ressources naturelles et des territoires. Or, d'une part, la formule contractuelle - qui prend sa source dans l'économie néo-institutionnelle- tend à extraire l'ensemble des rapports sociaux, des contextes et des déterminants sociologiques ou historiques, au profit d'une conception de relations qui associeraient librement des individus conscients et mus par leurs intérêts propres. D'autre part, cette formule contractuelle s'inscrit dans une vision normative de la régulation sociale que l'anthropologie questionne à l'examen des situations de terrain.

Et si notre analyse croisée de l'économie institutionnelle et de la socioanthropologie s'empare ici du contrat de gestion, elle traite cependant moins du rapport à la norme établie dans le contrat - à savoir son efficacité dans la " juste " répartition des richesses, des informations et des pouvoirs entre les contractants - qu'elle n'explore les changements sociaux induits par cette tentative de normalisation. Dans les discours et dans la pratique des acteurs du développement, le choix d'intervenir à l'échelle "locale " prend sa source dans " une idéologie très communautariste " (Bako-Arifari et Laurent, 1998, Olivier de Sardan 2010, Lavigne Delville 2006, Agrawal et Gibson 1999, Blaikie, 2006). En effet, du point de vue des bailleurs de fonds et des représentants d'organisations internationales ou non gouvernementales, l'échelle communautaire représente un espace de relations d'interconnaissances fortes qui serait, pour cette raison, plus à même de mobiliser les ressorts de l'action collective. Il s'agit là en vérité d'un double malentendu. D'une part, la communauté villageoise est, comme toute société, traversée par des intérêts divergents entre groupes sociaux et/ou statutaires (castes, lignages, groupes d'âges, sexes), des hiérarchies et des inégalités, et par des conflits, et ne forme pas un groupe homogène, loin s'en faut (Bako-Arifari et Laurent, 1998, Bardhan, 2002, Lavigne Delville et Hochet, 2005, Olivier de Sardan, 2013). D'autre part, les présupposés sousjacents à l'action collective prennent leur source dans une approche 
individualiste où le collectif s'appréhende comme un agrégat de stratégies d'acteurs atomisés dont les choix sont supposés rationnels et les comportements intentionnels, conscients et libres. Cette approche justifie la nécessité d'une coordination, ici de type contractualisé. Ce double malentendu ouvre la place à une approche croisée dans laquelle les dynamiques sociales à l'œuvre ne devraient pas être lues comme une addition mécanique des intentionnalités individuelles, mais pas non plus comme le seul produit des pesanteurs structurelles. Les stratégies des acteurs sont comme autant de choix en partie déterminés par les structures sociales, notamment lorsque les acteurs sont mobilisés dans les projets de gestion en commun des ressources qui les concernent au premier chef.

Nous verrons ces malentendus conceptuels à travers les décalages qui se sont opérés entre la mise en œuvre des dispositifs contractuels censés réduire les coûts de transaction, et les dynamiques sociales, les formes d'exclusion, de conflits et de concurrences sur les espaces et les ressources que de tels dispositifs ont pu engendrer ou exacerber.

Vers un cadre d'analyse des dispositifs contractuels de gestion des ressources

On voit toute la difficulté de s'emparer d'un tel objet d'étude, qui relève généralement de démarches fonctionnalistes, pour analyser les décalages de sens entre la logique des contrats et la pratique. II importe d'observer chacun des stades de la contractualisation pour comprendre les arguments qui sous-tendent les fonctions attribuées au contrat : (i) formaliser une relation entre des acteurs ; (ii) agréger des intérêts divers en vue d'une institutionnalisation; (iii) rechercher un équilibre entre des parties contractantes en asymétrie d'informations et de pouvoirs. Ces arguments sont mis à l'épreuve comme un cadre d'analyse qui nous permettra de comparer les processus de gestion contractuelle en œuvre au Mali, au Sénégal et au Niger, entre logiques utilitaristes et dynamiques de changement social.

Dans une première partie, nous aborderons la façon dont s'établissent, en préalable au contrat, les échanges d'informations sur les droits et sur les ressources, qui permettent de caractériser une situation initiale en vue d'atteindre un objectif supposé commun d'un milieu forestier géré durablement. Les différentes expériences montrent que cet objectif n'est, en effet, guère unique ni commun aux différents acteurs, et il se prête peu à 
un compromis sur les divergences (Mermet et al., 2006, Ishihara et Unai, 2009). Même en faisant l'hypothèse d'un accord sur un tel objectif, nous interrogeons la base sur laquelle se négocie la norme de gestion durable. Les cas étudiés permettront d'analyser des pratiques d'intervention (catégorisation des acteurs, délimitation des espaces et définition de techniques d'aménagement et de gestion des forêts) qui s'inscrivent dans une histoire longue des relations entre acteurs de la gestion des forêts en Afrique Subsaharienne.

Dans une seconde partie nous étudierons les négociations entre acteurs qui interrogent les arguments portant sur la diversité des intérêts. En effet, dans les discours du développement comme dans les arguments académiques mobilisés en faveur de la dévolution, le pluralisme constituerait un principe opérationnel préalable pour trouver, dans le processus, un accord entre les parties. Une grande partie des analyses ont ainsi recours à l'argument du pluralisme comme exigence minimale. Or cela participe d'un présupposé individualiste : les individus sont supposés libres de s'associer pour représenter les intérêts collectifs et être des interlocuteurs fiables. De fait, ce sont les groupes d'acteurs en jeu qui sont considérés comme des entités à la fois individuelles et équivalentes, c'est-à-dire qu'elles sont supposées avoir le même poids (politique ou économique) dans les décisions. Les diverses parties sont, en effet, considérées comme des entités homogènes et closes sur elles mêmes (par exemple les "villageois " en face des " exploitants forestiers privés » ou de "l'État »). Le risque de figer les acteurs dans les hiérarchies qui les structurent, et partant, de renforcer les acteurs dominants, est toujours présent.

Or, on voit se profiler une superposition d'institutions et d'autorités qui pourraient produire de l'anomie, provoquer des conflits là où ils étaient latents, ou d'en créer là où il n'y en avait pas (Boutinot et Diouf, 2006, Mbodj dans ce dossier). A moins que le contrat, susceptible de coordonner ce pluralisme, puisse ouvrir à des systèmes de subsidiarité ou de partage de responsabilités bien définis dans lesquels chacun trouve son compte et qui pourraient le légitimer. Les diverses expériences étudiées illustrent l'importance de considérer le processus d'élaboration des contrats pour interroger les modifications induites ou non sur les modes de gouvernance. En quoi ces démarches constituent-t-elles des forums de discussion, de négociations ou d'apprentissage collectif à la construction de nouvelles 
relations entre l'État et ses services publics, les élus locaux et les usagers et exploitants des ressources naturelles ? Ces points seront discutés dans une troisième partie.

\section{Les figures du contrat au Mali, au Niger et au Sénégal}

\section{L'historique de la gestion forestière}

II importe de rappeler que, malgré l'histoire des régimes politiques distincts que ces trois pays ont connu depuis les indépendances, la gestion de leurs ressources forestières demeure fondamentalement similaire et ce, sans solution de continuité depuis l'époque coloniale. Le premier code forestier colonial de l'Afrique Occidentale Française date de 1935. II répartit les forêts en deux catégories : les forêts qui relèvent du domaine privé de l'État (forêts classées) et celles qui sont protégées. Les populations riveraines ne disposent que des droits d'usufruit et les espaces forestiers demeurent sous le contrôle des services de l'État. Les agents forestiers sont, pour leur part, fidèles à la tradition coloniale et investis d'un pouvoir quasi absolu sur les ressources forestières; ils considèrent les populations villageoises comme le principal ennemi des forêts.

Ces rapports antagoniques sont reconduits par les indépendances. Partout la politique de l'époque coloniale reste appliquée à la faveur de la permanence des anciens administrateurs et/ou militaires coloniaux transformés en conseillers techniques.

Puis, d'une manière générale, les sécheresses qui secouent l'Afrique subsaharienne et les crises pétrolières des années 1970 et 1980 vont inviter les États à se préoccuper des questions d'environnement et de gestion forestière (Yamba, $2001: 145$ ) avec l'aide des institutions de coopération internationales. Ainsi, la Banque Mondiale initie ses interventions dans le domaine de l'environnement en élaborant des programmes énergétiques dès le début des années 1980, appuyés sur une crise " annoncée " du bois énergie, visant à assurer la consommation urbaine et lutter contre la déforestation (Anderson et al. 1985, Eckholm et al., 1984). Ceux-ci donnent lieu à une Revue des Politiques et Stratégies dans le Secteur des Energies Traditionnelles (RPTES) dans 5 pays du Sahel entre 1993 et 1995. Puis ils conduisent à l'établissement d'un programme pour « la gestion durable et participative des énergies traditionnelles et de substitution », appelé Projet 
Energie II au Niger (1989), Projet Energie Domestique au Mali (1996) ${ }^{1}$ et PROGEDE au Sénégal.

Parallèlement, les lois de la décentralisation, amorcées dès les années 70 au Sénégal (avec la création des communautés rurales en $1972^{2}$ ) et au Niger et dans les années 90 au Mali, établissent des brèches dans le système de monopole étatique de la gestion des forêts. Ainsi au Sénégal, en 1996, le principe de constitution et de gestion de domaines est formellement reconnu aux collectivités territoriales, ce qui n'est pas le cas au Niger et fonctionne de manière très peu opérationnelle au Mali. Le Sénégal se distingue également du Niger et du Mali dans la structure même de la filière du bois énergie. La filière connaît, dans ce pays, une longue histoire dominée par un oligopole puissant des exploitants forestiers du charbon du bois et fait l'objet, depuis la production jusqu'au commerce de détail, $d^{\prime}$ 'une politique particulière (Cf. Boutinot dans ce dossier). Pourtant, malgré ces différences, l'étude des trois cas montre certaines similitudes dans les démarches de transfert de gestion entreprises par les projets mis en œuvre depuis la fin des années 1990.

\section{Emergence des transferts de gestion}

L'exploitation forestière va reposer sur une évaluation des ressources en bois des bassins d'approvisionnement des grands centres urbains à l'aide de schémas d'aménagement qui sont des outils de planification territoriale et sectorielle. D'autre part, une innovation de taille est introduite avec la création d'une fiscalité sur le bois qui induit de nouveaux modes de prélèvement des ressources forestières et de répartition des revenus issus de son exploitation.

Au Mali, l'établissement de contrats entre l'État et les communautés rurales est concrétisé par la loi 95-003 portant organisation de l'exploitation, du transport et du commerce du bois. Cette loi stipule la création des marchés ruraux de bois gérés par des structures rurales de gestion (SRG). Sous la pression des donateurs, est aussi créée la Stratégie

1 Dotés de 11 M€ au Niger et 7,8 M€ au Mali.

2 La décentralisation ayant démarré bien avant en ce qui concerne les zones urbaines au Sénégal 
Energie Domestique (SED) chargée de la mise en œuvre de la réforme ${ }^{3}$ et largement inspirée de l'expérience nigérienne, considérée à cette époque comme une réussite (Noppen et al 2004 ; Djiré 2004, Kassibo 2010). Les forêts, quant à elles, changent de statut. Les forêts villageoises sont soumises à une délimitation et à un inventaire écologique, réalisés par des opérateurs privés, comme au Niger à partir des années 90 , mais elles ne sont pas encore aménagées. On les qualifie d'exploitations de type orienté. Les anciennes forêts classées de l'État, qui sont délimitées et aménagées, répondent, elles, au titre d'exploitation contrôlée. Enfin, les forêts qui ne font l'objet ni de délimitation, ni d'aménagement, sont qualifiées d'incontrôlées et sont directement placées sous le contrôle des agents forestiers qui délivrent des permis de coupe aux particuliers i.e. les exploitants privés. Les structures rurales de gestion (ou SRG) n'ont jamais pu bénéficier de la taxe d'exploitation prévue ni assurer son prélèvement, et sa répartition entre l'État et les autres partenaires, contrairement à la loi de 1995. En 1998, un décret (402 du 17 décembre 1998) met un terme définitif à cette velléité. Toute cette expérience qui prévoyait aussi la participation des communes au processus, s'est finalement déroulée avant leur mise en place effective ${ }^{4}$. Depuis leur entrée en scène officielle, aucun domaine forestier ne leur a été transféré.

Dans une facture similaire, au Sénégal, le statut de forêt communautaire est défini par la loi de décentralisation de 1996, qui prévoit des transferts de compétences aux collectivités locales ${ }^{5}$.Mais l'État reste garant des terres forestières ${ }^{6}$ dans la réforme de 1998 et délègue les compétences aux collectivités locales sur la base d'un protocole d'accord ou plan de gestion. Dans le cadre du Programme de Gestion Durable et Participative des Energies Traditionnelles et de Substitution (PROGEDE) ${ }^{7}$, neuf forêts

\footnotetext{
${ }^{3}$ Environ trois cents marchés ruraux de bois sont mis en place par cette structure avant sa disparition.

${ }^{4}$ Créées en 1996, les collectivités décentralisées du Mali ne sont devenues opérationnelles qu'avec les élections de 1999 qui ont mis en place leurs organes délibérants et exécutifs.

${ }^{5}$ Loi n ${ }^{\circ}$ 96-07 du 22 mars 1996 portant transfert de compétences aux Collectivités Locales et décret d'application $n^{\circ}$ 96-1134 du 24 décembre 1996.

${ }^{6}$ Celles-ci sont composées, selon l'article R.2, de « l'ensemble des zones classées comprenant les forêts classées, les réserves sylvo-pastorales, les périmètres de reboisement et de restauration, les parcs nationaux, les réserves naturelles intégrales et les réserves spéciales ».

${ }^{7}$ Crée en décembre 1997 par l'arrêté interministériel n 10291 - le PROGEDE vise à finaliser des plans d'aménagement dans les 2 arrondissements et à développer des activités permet-
} 
communautaires (381 $074 \mathrm{ha}$ ) ont été soumises à un plan d'aménagement et quelques 300 CVGD ont été créés. Ces forêts ont fait l'objet d'une charte de gestion et d'un code local spécifique à la production de charbon de bois.

Dans le cas du Niger, le transfert de gestion est établi au début des années 90, également au travers de projets successifs ${ }^{8}$. En 1992, une loi sectorielle (ici aussi forestière : ordonnance 92-037) y est également promulguée. Des marchés ruraux du bois énergie sont créés au niveau des villages riverains de zones forestières préalablement identifiées et soumises à quota d'exploitation. Ces marchés ruraux ont vocation à devenir les seuls points de commercialisation du bois issus de ces zones. A partir de 2000, ce sont, comme au Mali, des opérateurs privés qui fournissent l'appui à la création et au suivi de ces marchés ruraux (cf. Gautier et al. 2013). Les transferts attribuent aux villages riverains de droits nouveaux : exploitation du bois dans les massifs, commercialisation sur le marché rural et perception de la taxe liée sur le transport du bois commercialisé. Seuls les limites des massifs forestiers, le ou les villages concernés et la nature du marché rural sont formalisés. Au total, 180 marchés ruraux, dont $75 \%$ se situent à plus de $80 \mathrm{kms}$ de centres urbains et couvrent une superficie d'environ 560000 ha, sont recensés en 2003. 135 d'entre eux sont des marchés contrôlés (Noppen et al. 2004). La politique de décentralisation, menée à partir de 2004, permet par ailleurs l'émergence de communes dotées d'un conseil et d'un organe exécutif élu. Cependant, celles-ci ne disposent d'aucun pouvoir sur la gestion des ressources naturelles, sauf à obtenir de l'État des concessions rurales temporaires.

tant de diversifier les revenus villageois et de réduire la pression sur les ressources forestières (apiculture, intensification élevage, réserves fourragères, horticulture). Progede, Plan d'aménagement de la forêt communautaire de Saré Gardi Kolda, 2004, p.8

${ }^{8}$ Le projet Banque Mondiale Energie II (1989-1998) et le projet bilatéral Energie domestique (2000-2003), tous deux sous financement danois, s'appuient sur un zonage des déséquilibres potentiels entre offre et demande, réalisé à l'échelle des bassins d'approvisionnement des villes (schéma directeur d'approvisionnement). Puis en 2001, un projet BAD, le PAFN, a démarré avec une approche analogue. 


\section{Le rôle de la délimitation des acteurs et des territoires concernés par le contrat}

L'établissement des contrats visant le transfert de gestion des ressources forestières suppose d'identifier les personnes concernées (les parties) pour leur attribuer droits et obligations sur l'objet du contrat. Les exemples étudiés montrent que la délimitation des acteurs et des espaces s'effectue souvent indépendamment du contexte social. En effet, le choix du massif forestier est d'abord réalisé en fonction de critères écologiques, par délimitation à partir d'un survol aérien et sur inventaire des espèces, au Sénégal, mais également en fonction de la proximité par rapport aux centres urbains au Mali et au Niger. Des droits d'accès et d'utilisation des ressources, le plus souvent exclusifs et collectifs, sont attribués à des groupes sociaux donnés, dont la composition varie selon les cas. Au Niger, c'est le service de l'Environnement qui procède à la création des Structures Locales de Gestion (SLG), dans les villages riverains des forêts délimitées. Au Mali, c'est la Cellule Combustible Ligneux (CCL), organe de tutelle qui est chargé par la SED de la constitution des Structures Rurales de Gestion (SRG), sur le même modèle. Au Sénégal, les agents du service forestier, recrutés dans le PROGEDE, mettent en place des Comités Villageois de Gestion et de Développement (CVGD) à l'échelle des villages incorporés dans l'espace forestier soumis à aménagement, lesquels sont rassemblés en un Comité Intervillageois (CIV) à l'échelle de la forêt. II arrive, notamment dans le cas de la forêt de Saré Gardi que nous avons plus particulièrement étudiée, que l'espace délimité déborde des limites administratives de la communauté rurale, et empiète sur la collectivité locale voisine. Les transferts de gestion sont souvent suscités et initiés pour répondre à des exigences d'ordre technique et non à celles des institutions dont dépendent localement la gestion des ressources et les conditions de vie des populations. Ce qui ne manque pas de peser lourdement sur leur mode de fonctionnement.

Dans les trois pays étudiés, le "massif » forestier constitue donc l'échelle de gestion. Sa délimitation est réalisée à partir de critères techniques et d'inventaires de la ressource et non à partir des liens sociaux entre villages et communautés, s'écartant ainsi de la définition d'un bien patrimonial comme préalable au contrat. Les communautés ou les échelles choisies posent alors le problème de la légitimité des autorités entre les chefs traditionnels et les élus locaux. Le pouvoir traditionnel y est 
concurrencé au Mali, réintroduit au Sénégal à la tête des comités villageois, et représente une entité polarisée par le marché local au Niger. Des problèmes de cohérence avec la décentralisation politique en sont souvent la conséquence et apparaissent déjà lors de l'élaboration du contrat.

\section{Mise en place et négociation du contrat}

Ces démarches souvent "suscitées " de l'extérieur dans l'objectif de promouvoir la gestion décentralisée des forêts et d'engager une lutte contre la pauvreté au travers de la fourniture d'énergie, posent la question de la pluralité des acteurs, des institutions concernées et des intérêts représentés. La faible articulation entre le diagnostic préalable au transfert de gestion et la structuration sociale, est source d'exclusion, de conflits et a des conséquences sur les processus d'élaboration du contrat. On retrouve ce décalage dans les négociations.

\section{Une faible articulation diagnostic-structuration sociale}

Les diagnostics villageois, bien que basés sur des études techniques et des enquêtes, n'ont pas mis au jour l'aspect sociologique des problèmes. Dans les trois pays, le processus d'élaboration a donc posé la question de l'échelle sociale, dite communautaire, et celle de la constitution, préalable à toutes négociations, de comités ad hoc qui entérinent la dévolution.

Au Mali, par exemple, la dévolution des droits de gestion est apparue, aux yeux des habitants, comme la rétrocession d'un bien communautaire confisqué depuis la période coloniale. Pour les villageois, ce bien ne devait pas faire l'objet d'un monopole de la part d'une partie de la communauté, en l'occurrence celle des bûcherons. Mais, dans la commune de Siby, les bûcherons villageois ont exclu les exploitants étrangers des SRG, dès leur constitution et ils ont fini par les expulser du terroir, au nom de l'autochtonie $^{9}$ (voir les articles de Keita et Mbodj dans ce même ouvrage).

9 Dans un autre cas, analysé par Gautier et al. (2006) le clan des étrangers s'est opposé à celui des autochtones et a abouti à la scission du marché rural. 
Au Sénégal, les animateurs du PROGEDE ont procédé, dès 1999, à des diagnostics participatifs dans les villages polarisés par la forêt ${ }^{10}$. La bonne volonté des populations à jouer le jeu a tenu au fait qu'un tel projet, à l'instar de tant d'autres, restait une opportunité pour elles d'améliorer leurs conditions d'existence mais on ne peut, dans ce cas, parler d'appropriation par les villageois des objectifs du projet. Les diagnostics participatifs menés avaient également pour but de réunir les populations en assemblées afin de constituer des comités de gestion. Ces derniers étaient présentés comme " des intermédiaires entre le PROGEDE et les populations " et les chefs de village ou leurs proches parents ont été " naturellement " choisis pour les représenter. Cette démarche présente une situation assez commune à nombre de projets de développement dans lesquels on observe la mise en œuvre simultanée de diagnostics villageois et de comités de gestion. Alors que les premiers sont censés sonder les attentes des populations locales, les seconds sont déjà une réponse organisationnelle aux actions futures. Le diagnostic n'établit pas une réelle situation de référence pour les acteurs. Et ces comités deviennent, pour le cas du Sénégal, des institutions locales susceptibles de jouir d'une autorité qui entre en concurrence avec celle des élus locaux qui forment le conseil rural. En ce sens, on est en droit de se demander si le diagnostic constitue un mode de connaissance et de partage des informations sur le futur projet, voire d'échanges sur les attentes des uns et des autres, ou bien s'il est une vision normative imposée qui permet aux projets de créer la demande, de justifier leurs interventions et de les légitimer.

Au Niger, d'une manière similaire, le choix des massifs forestiers inclus dans la gestion contractuelle ne fait généralement pas suite à une demande des communautés villageoises concernées, même si ce cas a pu être parfois signalé. Le diagnostic préalable, très encadré au niveau technique, ne prévoit pas de négociation avec les villageois par rapport aux règles de fonctionnement des marchés ruraux, ni d'appui organisationnel,

10 Les comptes rendus et la synthèse des MARP (Méthodes Accélérées de Recherche Participative) de Saré Gardi, montrent que la demande sociale des populations ne correspond pas toujours aux propositions du PROGEDE. En effet, les attentes de la population sont traduites sous une forme de liste de priorités où l'accès à l'eau est la priorité $n^{\circ} 1$ et l'aménagement de la forêt la priorité $n^{\circ} 7$ (Boutinot, 2004). 
une fois le marché créé. En fait, le processus de création des marchés ruraux au Niger a surtout été marqué par une forte opposition des services en charge de la politique forestière et des commerçants urbains de bois énergie, auparavant détenteurs des permis d'exploitation des forêts. La négociation a donc davantage concerné les commerçants urbains qui avaient entamé un blocage de la réforme. Ce processus rend ainsi compte d'un marchandage entre des acteurs qui disposent d'un pouvoir important sur les revenus de l'exploitation du bois énergie (administration forestière et commerçants de la filière bois) et non d'une négociation entre les différents intérêts, incluant ceux des villageois usagers des massifs forestiers.

\section{Négociation et enjeux des contrats}

Les enjeux sont liés à la répartition des activités, des revenus et des taxes, entre comités ad hoc, collectivités locales, services forestiers et acteurs privés, en tenant compte des nouveaux acteurs introduits par les réformes. Les contrats révèlent plusieurs aspects des relations sociales existantes dans la gestion des forêts sous aménagement. Ils servent à spécifier les règles, les droits et les devoirs des parties au contrat à partir d'un niveau d'exploitation fixé par l'administration forestière. Ils structurent les communautés en désignant les acteurs clés de l'approvisionnement des marchés de bois et de la gestion environnementale.

Au Mali, contrairement aux SLG du Niger dont l'expérience malienne s'est pourtant fortement inspirée, les SRG ont été privées d'une part importante de leurs prérogatives. Elles doivent d'abord verser au service forestier les taxes d'exploitation du bois ou du charbon correspondant à leurs quotas pour bénéficier d'un droit de vendre (matérialisé au Mali comme au Niger par des coupons) sur les marchés. De plus, le service de la Conservation de la Nature perçoit des taxes qui ne sont pas redistribuées aux SRG comme il était prévu. Dans le domaine de la répartition des recettes fiscales on assiste en fait à un véritable imbroglio juridique de la part des décideurs institutionnels (Ministère de l'environnement et de l'assainissement et Direction nationale de la conservation de la nature) qui se décline, de 1995 à 2004, à coups de promulgation et d'abrogation de décrets. L'absence de clé de répartition des taxes entre les trois niveaux de collectivités territoriales (région, cercle, commune) pose problème pour leur allocation. De plus, les trois types d'exploitation forestière (orienté, 
contrôlé, incontrôlé) qui déterminent le montant des taxes à payer selon l'origine du bois et/ou du charbon, n'ont pas de réalité sur le terrain. Ceci a constitué un véritable problème pour les agents forestiers chargés d'appliquer le décret de 2004. Ces derniers ont donc continué à appliquer le décret 98-402 - abrogé -, en espérant la parution d'un arrêté plus explicite sur la démarche à suivre. De son côté, la commune participe à la fixation des quotas annuels d'exploitation mais elle n'est impliquée ni dans la création des marchés ruraux, ni dans l'exploitation des ressources forestières. Elle n'est pas non plus partie prenante dans la signature du contrat liant la SRG à la Cellule Combustible Ligneux. Elle bénéficie d'une part infime des ristournes de la part de l'État qui, de surcroît, comme au Sénégal et au Niger, ne doivent servir qu'à la réalisation d'actions environnementales (pépinières, reboisement, pare feux, etc.).

Au Sénégal, il apparaît une réelle distinction entre ceux qui produisent du charbon de bois et ceux qui sont censés entretenir la ressource... pour les autres. Ainsi, le contrat local est composé, d'une part, d'une charte assez générale qui réglemente les espaces d'usufruit via l'accès aux ressources (produits forestiers, espaces de conservation ou pâturage en forêt) et les interdictions ${ }^{11}$ et, d'autre part, d'un code précisant les modalités du commerce du charbon de bois. La charte est signée par les chefs de village, les deux présidents des communautés rurales concernées, les services forestiers et le sous-préfet. Un comité inter-villageois (CIVGD) est chargé de contrôler les pratiques et les actes des contrevenants stipulés dans la charte générale. En revanche, le code relatif au charbon de bois a été signé par les présidents des comités villageois, les présidents des deux communautés rurales et les patrons charbonniers, sous l'égide des services forestiers.

Il existe donc au Sénégal une distinction entre l'usage domestique, pour lequel les autorisations sont inscrites dans la charte, et l'exploitation à

11 Le code ne précise d'ailleurs pas les zones de terroirs où sont permis les défrichements agricoles, ce qui laisse supposer que tout défrichement est impossible et pose la question de la reconstitution biologique des terres agricoles. Tous les feux de brousse sont aussi interdits. 
finalité commerciale, celle du charbon, pour laquelle une procédure complexe est mise en place, sous le contrôle des CVGD ${ }^{12}$.

Une clef de répartition des recettes de la vente prévoit que $25 \%$ du prix du sac de charbon soit théoriquement prélevé sur la production des patrons charbonniers contractuels venant de l'extérieur au bénéfice des institutions locales (Conseil Rural, CVGD, CIVGD). Ce code particulier révèle à la fois des relations économiques plus précises entre les acteurs et des rapports de force plus stricts entre les producteurs villageois et les grands patrons charbonniers de Dakar qui veulent maintenir leur oligopole sur la filière. Mais en cas de non respect des règles fixées dans le contrat, le code local ne précise ni les acteurs autorisés, ni les modalités de suivi des infractions, ni les procédures de recouvrement. Toutefois, les textes de loi de la décentralisation octroient aux Conseils Ruraux un droit explicite de contrôle sur la gestion des fonds et des comptes des comités villageois. En dupliquant ainsi les responsabilités et en ne les précisant pas, cette contractualisation favorise les conflits d'autorité sur la répartition des ressources issues de la production forestière. Des conflits que nous avons constatés entre les chefs de CVGD et les présidents de conseils ruraux au sujet des autorisations de coupe délivrés par une autorité à l'insu de l'autre (CVGD-Conseil Rural, et réciproquement), relèvent directement de ce pluralisme institutionnel mis en place par le projet. Lequel a d'ailleurs favorisé des malversations qui ont pu discréditer certains de ces comités villageois, ainsi que des agents forestiers (voir Blundo, dans ce même numéro).

Au Niger, les règles liées au fonctionnement du marché rural montrent une innovation majeure par rapport aux autres cas étudiés. En effet, la SLG perçoit à la source, directement sur le lieu du marché rural, la taxe de transport payée par les commerçants (qui remplace le permis de coupe payé aux services forestiers ${ }^{13}$ ). Ensuite, une partie du montant reste à la

12 Les autorisations sont données par les comités villageois, puis transmises aux services forestiers pour qu'un permis d'exploiter soit établi.

13 Le permis de coupe reste valable pour les achats de bois par les commerçants sur d'autres zones que celles rattachées à des marchés ruraux. Sur ces zones dites incontrôlées, les communautés villageoises ne perçoivent ni revenus quand les 
disposition de la SLG, avant que le reste ne soit rétrocédé. Mais les contrats sont, au Niger, très encadrés par des dispositifs législatifs et réglementaires (ordonnance de 1992 et de nombreux arrêtés) et peu donnent lieu à une réelle négociation contractuelle sur le plan de gestion, les quotas, les taxes et leur répartition. Le niveau d'exploitation est ainsi déterminé par des quotas annuels collectifs, fixés par les projets et l'administration forestière, en fonction de la nature du marché rural ("contrôlé " ou "orienté ") ${ }^{14}$. Les quotas ne sont pas négociables. Cependant, même si l'accès aux forêts délimitées et l'exploitation du bois énergie sont théoriquement réservés aux bûcherons accrédités par la SLG, le pouvoir de négociation face aux commerçants reste assez faible pour la fixation des prix, pour le versement des taxes. Le reçu de la taxe est un justificatif qui indique le marché rural de provenance du bois commercialisé ${ }^{15}$. C'est, en dernière instance, l'administration des forêts qui est chargée du contrôle du dispositif, souvent décrit dans les enquêtes en termes d'abus de droits, de répression, voire de prélèvements illicites dans les stocks de bois ou dans les caisses des marchés ruraux. Les sanctions qui sont imposées ont donc une dimension collective.

Le tableau 3, ci-après, présente une synthèse des différents cas étudiés.

commerçants exploitent le bois des massifs riverains de leurs villages, ni part du montant des permis de coupe.

${ }^{14}$ Les deux types sont fonction de l'état de la ressource et de la distance au centre de consommation. Le "marché contrôlé ", est assorti d'un plan de gestion de la forêt prévoyant des parcelles et des quotas annuels de coupe fixés pour une durée de 4 à 8 ans. Le marché orienté», sans plan de gestion est limité à la collecte de quotas de bois mort.

${ }^{15}$ Un marché noir de coupons de transport a pu ainsi être signalé sur quelques sites. Il sert à commercialiser du bois d'un marché rural avec les coupons d'un autre marché rural à taxation inférieure. 


\begin{tabular}{|c|c|c|c|}
\hline & $\begin{array}{l}\text { Sénégal (Sare } \\
\text { Gardi) }\end{array}$ & $\begin{array}{l}\text { Mali (Bassin } \\
\text { d'approvisionnement } \\
\text { de Bamako) }\end{array}$ & $\begin{array}{l}\text { Niger (Bassin } \\
\text { d'approvisionnemen } \\
\text { t de Niamey) }\end{array}$ \\
\hline $\begin{array}{l}\text { Situation } \\
\text { initiale et } \\
\text { base du } \\
\text { contrat : } \\
\text { choix de } \\
\text { l'objet du } \\
\text { contrat et } \\
\text { des co- } \\
\text { contrac- } \\
\text { tants }\end{array}$ & $\begin{array}{l}\text { La loi forestière } \\
\text { de } 1998 \text { impose } \\
\text { un plan } \\
\text { d'aménagement } \\
\text { pour les forêts } \\
\text { communautaires ; } \\
\text { Délimitation } \\
\text { technique et } \\
\text { écologique par } \\
\text { projets ; } \\
\text { Diagnostic pour } \\
\text { organisation en } \\
\text { CVGD. }\end{array}$ & $\begin{array}{l}\text { La loi forestière et les } \\
\text { décrets définissent } \\
\text { les conditions } \\
\text { d'exploitation du } \text { transport et du com- } \\
\text { merce du bois (sur le } \\
\text { modèle du Niger); } \\
\text { Diagnostic général } \\
\text { Schéma Directeur } \\
\text { d'Approvisionnement } \text { (SDA') à l'échelle du } \\
\text { Bassin d'approvision- } \\
\text { nement ; } \\
\text { Plan d'aménagement } \\
\text { et diagnostic villa- } \\
\text { geois déterminant le } \\
\text { lieu d'implantation du } \\
\text { marché. }\end{array}$ & $\begin{array}{l}\text { Antériorité du pro- } \\
\text { jet : les dispositifs et } \\
\text { la démarche de } \\
\text { projet sont repris } \\
\text { dans les lois et dé- } \\
\text { crets. } \\
\text { Diagnostic général } \\
\text { (SDA) à l'échelle du } \\
\text { bassin } \\
\text { d'approvisionnemen } \\
\text { t des villes et délimi- } \\
\text { tation de massifs } \\
\text { selon offre de bois et } \\
\text { distance ; } \\
\text { Identification des } \\
\text { villages riverains et } \\
\text { limites de la forêt } \\
\text { dont la gestion est } \\
\text { transférée. }\end{array}$ \\
\hline $\begin{array}{l}\text { Prise en } \\
\text { compte de } \\
\text { la diversité } \\
\text { des intérêts } \\
\text { dans } \\
\text { l'élaboratio } \\
\text { n des con- } \\
\text { trats }\end{array}$ & $\begin{array}{l}\text { Diagnostic parti- } \\
\text { cipatif et constitu- } \\
\text { tion des comités } \\
\text { ad hoc simulta- } \\
\text { nément ; } \\
\text { Contrat organisé } \\
\text { autour du plan de } \\
\text { gestion du char- } \\
\text { bon; }\end{array}$ & $\begin{array}{l}\text { Application d'une } \\
\text { méthodologie forma- } \\
\text { lisée de création de } \\
\text { marchés ruraux de } \\
\text { bois énergie avec rôle } \\
\text { des bureaux d'études, } \\
\text { comme au Niger ; } \\
\text { Constitution du dos- } \\
\text { sier d'agrément par le } \\
\text { village pour consti- } \\
\text { tution d'une SRG ; } \\
\text { Limites floues de la } \\
\text { SRG entre autorités }\end{array}$ & $\begin{array}{l}\text { Processus technique } \\
\text { de création de mar- } \\
\text { ché rural ; } \\
\text { Faible lien entre SLG } \\
\text { et les assemblées } \\
\text { villageoises } \\
\text { d'usagers de la zone } \\
\text { forestière; }\end{array}$ \\
\hline
\end{tabular}




\begin{tabular}{|c|c|c|c|}
\hline & $\begin{array}{l}\text { producteur char- } \\
\text { bon de bois/ } \\
\text { contractuel ; } \\
\text { Divers niveaux de } \\
\text { négocia- } \\
\text { tion (code/charte) } \\
\text { : commerçants } \\
\text { intégrés dans la } \\
\text { négociation éco- } \\
\text { nomique du code. }\end{array}$ & $\begin{array}{l}\text { communales et villa- } \\
\text { geoises (Qui fait } \\
\text { partie de la SRG ?). }\end{array}$ & $\begin{array}{l}\text { Limites de négocia- } \\
\text { tion face au pouvoir } \\
\text { des commerçants; } \\
\text { Remobilisation des } \\
\text { élites locales? }\end{array}$ \\
\hline $\begin{array}{l}\text { Réparti- } \\
\text { tion des } \\
\text { pouvoirs : } \\
\text { obstacles } \\
\text { au contrat } \\
\text { et conflits }\end{array}$ & $\begin{array}{l}\text { Confusion des } \\
\text { pouvoirs entre } \\
\text { conseils ruraux } \\
\text { élus (loi de dé- } \\
\text { centralisation } \\
\text { 1996) et comi- } \\
\text { tés villageois } \\
\text { nommés par les } \\
\text { forestiers ; } \\
\text { Confusions et } \\
\text { conflits portent } \\
\text { sur : la surveil- } \\
\text { lance ; la percep- } \\
\text { tion des taxes, la } \\
\text { délivrance autori- } \\
\text { sation de coupe, } \\
\text { les sanctions, les } \\
\text { dénonciations. }\end{array}$ & $\begin{array}{l}\text { Droits d'exploitation } \\
\text { prioritaires aux rési- } \\
\text { dents et non inclu- } \\
\text { sifs (accès limité des } \\
\text { massifs aux étrangers } \\
\text { et transhumants) ; } \\
\text { Revendications terri- } \\
\text { toriales des villageois } \\
\text { et application d'une } \\
\text { taxation illicite sur les } \\
\text { étrangers; } \\
\text { Commune non impli- } \\
\text { quée dans le proces- } \\
\text { sus de gestion et } \\
\text { dépourvue de do- } \\
\text { maine forestier (res- } \\
\text { ponsabilité de gestion } \\
\text { pas encore transfé- } \\
\text { rée comme prévu } \\
\text { dans Loi décentralisa- } \\
\text { tion } 2004 \text {. }\end{array}$ & $\begin{array}{l}\text { Règles d'accès visant } \\
\text { à être des incitations } \\
\text { aux communautés } \\
\text { en termes de reve- } \\
\text { nus du bois et de } \\
\text { perception d'une } \\
\text { part des taxes; } \\
\text { Mais dépendent de } \\
\text { la capacité des } \\
\text { communautés } \\
\text { locales comme des } \\
\text { communes à faire } \\
\text { respecter leurs } \\
\text { droits. }\end{array}$ \\
\hline
\end{tabular}

Tableau2 : Synthèse des cas étudiés

Ainsi, que ce soit au Mali, au Sénégal ou au Niger, les administrations forestières trouvent, à travers les communautés villageoises, le relais dont elles ont besoin pour mettre en place les politiques publiques de l'environnement et, notamment, pour organiser les aménagements fores- 
tiers ou prélever les taxes et obtenir une information sur une activité (l'exploitation du bois énergie) qui était peu visible auparavant. En échange, des incitations données aux communautés villageoises dépendent fortement de la capacité de celles ci à se faire représenter, à négocier et à faire respecter les règles inscrites dans le contrat. De plus, les dispositifs sont mis en place de façon ad hoc dans les différents pays pour contrôler la décentralisation. Ils affaiblissent, de ce fait, le pouvoir censé être attribué aux collectivités locales.

\section{Discussion : les contrats un vecteur de changement?}

Si l'on peut noter une certaine irréversibilité des changements dans la gestion des forêts et dans les dialogues entre acteurs, même partiellement engagés, on peut s'interroger en pratique sur le transfert de légitimité et d'autorité, à travers ces contrats. Une autre interrogation, sur laquelle nous nous arrêterons ensuite concerne les pouvoirs que ces contrats produisent, en tant que lieu alternatifs de décision.

\section{Quelle légitimité des institutions à travers les contrats?}

Deux types de légitimité sont à considérer. D'une part, une légitimité externe, entre les différentes institutions de la dévolution (CVGD, SLG, $S R G$ ), de la décentralisation administrative (collectivités locales) et de la déconcentration; et d'autre part, une légitimité interne, au sein de la communauté locale concernée par le transfert. Cette analyse tente de rendre compte du potentiel de changement produit par la gouvernance non-hiérarchique (Hufty, 2007) dans les rapports sociaux et politiques.

\section{La légitimité entre institutions ou légitimité externe}

L'élaboration des contrats de gestion rend compte des relations entre les comités de gestion, les collectivités territoriales issues de la décentralisation et l'État, à travers le service des Eaux et Forêts. Nous avons vu que les comités de gestion (CVGD au Sénégal, SRG au Mali, SLG au Niger) sont définis à une échelle micro locale, c'est-à-dire en deçà des collectivités territoriales élues. Dans plusieurs pays, ils sont dotés d'un statut de type associatif mais les compétences qui leur sont octroyées sont plus larges que celles du 
monde associatif en général et plus restreintes que celles des collectivités décentralisées. Ceci se traduit par des niveaux de décision imbriqués et des conflits d'autorité. Les comités de gestion se présentent comme concurrents face à d'autres institutions locales chargées de la gestion des ressources (d'autant plus lorsque plusieurs projets d'ONG de gestion des ressources forestières sont actifs au même endroit). De plus, dans tous les cas étudiés, la légitimité juridique des " comités " conçus spécifiquement pour l'application du contrat pose problème, dès lors que leurs compétences se superposent à celles des élus locaux. Si bien que non seulement la structuration des villageois en comités vient dupliquer les lieux de décision sur la gestion des forêts, mais elle les chevauche et les déplace. Contrairement aux arguments de Thomson (2000) selon lesquels le partage d'un même espace de ressources (forêts; pêcheries), entre différentes juridictions ou limites administratives, favoriserait l'action collective, nos exemples montrent que l'autorité sur la ressource se partage difficilement.

Ce sont les services forestiers déconcentrés qui bénéficient de cette imprécision et de la confusion des autorités dans le partage des produits de la vente, et la captation des taxes, des amendes et des confiscations. Ainsi au Sénégal, dans le massif forestier étudié composé à $55 \%$ de bois $d^{\prime} œ u v r e$, ce flou $n^{\prime}$ est pas neutre ${ }^{16}$. Le code local, établi à une échelle micro-locale, ne permet pas aux deux collectivités locales élues d'identifier la part qui leur revient, ni de les utiliser pour autre chose que des actions de gestion des ressources (c'est-à-dire pour des actions que les services forestiers n'ont plus les moyens en personnel humain et financier de réaliser). Toutefois ce système a montré ses limites dès lors que les conflits sont devenus trop fréquents et, les malversations aidant, les deux collectivités locales qui se partageaient la même forêt aménagée ont pu, à terme, obtenir gain de cause dans leur volonté de scinder le comité intervillageois et de détenir chacun le leur, ainsi qu'un quota spécifique de production de charbon.

La faiblesse de la légitimité externe est aussi illustrée par l'imprécision juridique des Comités villageois (CVGD, SLG, SRG). Elle ne leur permet pas d'engager des recours en cas de non respect du contrat par une autre des

16 Dans le code forestier au Sénégal, les recettes des amendes et confiscations sont affectées à $70 \%$ aux communautés rurales (CR) et à $30 \%$ aux services forestiers (dont $10 \%$ pour le dénonciateur et $20 \%$ pour le verbalisateur). 
parties prenantes comme au Niger. Au Sénégal, malgré la délégation de gestion finalement signée par les Conseils Ruraux élus, le statut juridique des " comités villageois " n'est pas avéré. Et au Mali, les SRG ont été déboutées de la collecte et de la répartition des taxes. Ces institutions locales ont donc un rôle très faible quant aux décisions relatives à la gestion des ressources forestières qui relèvent encore des services forestiers (normes d'exploitation et quotas fixés, contrôle et sanctions). Les amendements comme les recours contre les décisions ne sont pas suivis d'effets, et les dispositifs contractuels ne sont modifiables ou aliénables que du seul fait de l'État. Ces institutions locales restent, de fait, limitées dans leur potentiel d'empowerment des populations et dans leur dimension politique, pourtant nécessaire à la responsabilisation des villageois. On peut penser qu'elles ont surtout un rôle d'apprentissage des pratiques forestières ${ }^{17}$ et de structuration du monde rural dans des formes opérationnelles susceptibles de mobiliser les villageois et de leur apprendre les enjeux que devrait représenter pour eux la gestion des ressources.

\section{Le rôle ambigu des comités de gestion dans la gouvernance locale (légitimité interne)}

L'ambiguïté des comités de gestion se pose à la fois en termes de représentation des usagers locaux et en termes de relations ou de normes sociales qu'ils reproduisent.

Dans les trois pays, en ce qui concerne le choix des membres des comités, on n'est pas loin de membres élus par consensus ${ }^{18}$, ce qui fait apparaître le caractère contradictoire entre l'élection, idéalement à bulletin secret, et le consensus, à main levée. Dans de nombreux cas, les chefs de village et leur famille proche constituent les bureaux de ces comités qui concentrent les pouvoirs de décision et de gestion micro locales et sont susceptibles de bénéficier des appuis matériels et en formation que procurent la dévolu-

${ }^{17}$ Apprentissage à la carbonisation au Sénégal, aux plantations et au commerce du bois au Niger, au commerce mais aussi à la surveillance et au contrôle dans les trois pays.

${ }^{18}$ Comme revendiqué par exemple dans un document d'évaluation du projet PROGEDE au Sénégal Rapport de l'Evaluation Rapide Organisationnelle (ERO) de l'Ong FODDE, 2002, p 3. 
tion. Mais dans d'autres cas on peut observer la marginalisation de ces autorités coutumières comme signalé par Bene (2009). De toutes les façons, le rôle de chacun dans les bureaux est rarement spécifié et parfois peu compris de ses membres eux-mêmes, sauf le titre et la fonction du président. Ces rôles ne font d'ailleurs pas toujours l'objet d'un investissement réel. Ainsi, au Sénégal, le choix des membres des CVGD a été laissé à l'initiative des chefs de village et a entériné des rapports sociaux existants en renforçant l'autorité coutumière qui reste considérée, notamment par les porteurs du projet de la Banque mondiale, comme légitime. A l'échelle inter-villageoise, les CIVGD ont, en revanche, une légitimité fragile en tant que structure fédérative. Au Niger, la SLG du marché rural est censée représenter les villageois usagers des forêts. Comme au Sénégal, elle constitue l'interface avec les services forestiers et accrédite les bûcherons appartenant à la communauté villageoise. Mais contrairement eu Sénégal, la faible communication et la méfiance sont symptomatiques des relations entre les assemblées villageoises et les SLG qui en sont pourtant issues (Noppen et al. 2004, Olivier de Sardan, 2010). Par ailleurs, certains usagers des forêts, membres de droits des SLG, y sont peu ou faiblement représentés (femmes, éleveurs), ce qui peut apparaître comme un certaine délégitimation de ces groupes sociaux. Les enjeux de réappropriation des ressources multiplient " les sources de revendication de la légitimité sur les ressources et les territoires » (Gautier et al. $2008: .77$ ).

En ce qui concerne la participation des villageois, la gouvernance interne, issue du fonctionnement de ces comités, tend à produire, renforcer ou reproduire des relations de dépendance et/ou de hiérarchie préexistantes. Si dans le cas du Sénégal, tous les villageois sont membres d'office du comité, le document du plan d'aménagement précise que le comité regroupe les "volontaires " pour participer aux activités de développement du village. Mais il apparaît, pour les populations autochtones, un risque de laisser certains postes du CVGD à des populations allochtones mêmes si ces dernières sont très dynamiques dans les activités de développement (i.e. production de charbon de bois). D'où des oppositions entre les bureaux des CVGD (qui représentent l'autorité villageoise) et certains groupes "dynamiques» du village (en dehors du bureau). Ces groupes sont susceptibles de produire soit une cohabitation, dans le meilleur des cas, soit une opposition, dans le pire. 
Ainsi, ces institutions locales, créées dans l'espace de la dévolution ne favorisent pas la coordination mais sont plus souvent source de conflits ou de démobilisation des populations villageoises, ce qui affecte notamment les activités d'entretien de la ressource commune (entretiens de pare-feux et de lutte contre les feux de brousse, pépinières, etc.) et fait obstacle à la juste répartition de revenus ruraux.

\section{Les contrats comme producteurs de pouvoir :}

le rôle de la participation et du capital social

Les dispositifs contractuels étudiés dans le cas des forêts illustrent les enjeux qui se posent dans la gouvernance. Les arguments qui soutiennent l'intérêt du contrat font référence à la participation des populations villageoises, sollicitée dans tous les projets de développement ou de gestion des ressources que nous avons observés. Cette participation renvoie, entre autres, à la lutte contre la pauvreté et à la volonté de prendre en considération les intérêts des acteurs qui vivent directement de ces ressources et dont les conditions de vie dépendent. Si la démarche est louable en soi, elle reste bien souvent contrainte dans la difficulté à trouver l'échelle adéquate pour stabiliser une action collective. Ainsi, on peut s'interroger sur la capacité de ces contrats à être ou à devenir des lieux alternatifs aux politiques publiques imposées face aux règles du jeu des acteurs à la base.

L'observation des dispositifs contractuels montre comment la dialectique entre les règles imposées par les réformes et la production de nouvelles règles du jeu s'incarne dans deux directions. Ces dispositifs jouent à la fois un rôle de relais des services forestiers et ils illustrent les tensions dans la politique de décentralisation. L'échelle de la dévolution a été choisie dans les réformes forestières sur les critères techniques, organisationnels et écologiques utiles à l'aménagement d'une forêt ou d'un espace de production (schéma directeur de la région de Niamey au Niger ou de Bamako au Mali pour la production de charbon et de bois). La difficulté réside dans l'articulation des intérêts entre une pluralité d'acteurs et dans la forme de gouvernance qu'elle impose. On est souvent loin des présupposés théoriques de l'action collective rendant compte d'arrangements entre des protagonistes orientés vers un même objectif. Le paradoxe étant que ce sont ceux-là même qui produisent du pluralisme local entre diverses 
autorités de nature différente qui imposent la nécessité d'un arbitrage par le contrat.

Nous avons pu aussi observer que les règles établies au travers les contrats locaux avec l'incertitude juridique qui les accompagne, renforcent les politiques sectorielles qui entraient déjà en contradiction avec la politique de décentralisation. Les conventions locales à l'échelle villageoise permettent au pouvoir central de maintenir son contrôle sur l'espace ou sur les ressources financières (taxes) qui reviennent de droit aux collectivités locales dans le cadre de la décentralisation. Cette tension est notamment visible au travers de la redistribution des ressources financières et des taxes issues des forêts. Seule une part réduite des fonds collectés par les structures de gestion locales arrive jusqu'aux collectivités locales (Noppen et al. 2004 ; Kassibo 2010). Cela conduit à une prise en charge accrue par les villages des services de base qui relevaient auparavant de l'État sans empêcher des prélèvements indus des services forestiers sur les fonds que ces structures quand celles ci ont pu en constituer (Noppen et al. 2004). Cette redistribution imparfaite des ressources constitue donc un facteur de risque qui peut obérer le fonctionnement des collectivités locales (communes, ou communautés rurales) mises en place dans la décentralisation. Dans tous les cas, cette confrontation entre les règles imposées et les pratiques se fait alors au détriment d'une légitimité de la décision locale, en dépit du discours sur la participation.

Une faible adéquation est observée entre organisation de comités ad hoc promue par les contrats, les échelles de décision politique et les pratiques locales antérieures et contemporaines de la gestion des forêts. Bien souvent, elle favorise des processus d'inclusion / exclusion des populations sous la dénomination parfois très explicite de "résidents " et "d'étrangers ". Dès lors que les délimitations d'une forêt, d'une autorité locale, d'une pratique sociale se superposent, la question de la représentation des populations dans les instances de décisions est complexe, multiple, confuse. Elle pose de façon aigue la question de la redevabilité. Ces dispositifs contractuels ne sont pas construits par rapport à l'espace public de la citoyenneté. II n'est donc pas aisé de rendre compte de la redevabilité des bénéficiaires des transferts de pouvoir dans ce contexte, dès lors que la multiplication des institutions locales tend à diluer les responsabilités, à les affaiblir et, partant à favoriser les pratiques corruptives (Polanyi, 1983 :169). Ainsi, les comités de gestion en charge de l'application de ces 
conventions sont investis de fonctions nouvelles et, dans le cas du Sénégal, ces fonctions, vont jusqu'à surpasser celles des représentants élus d'un conseil rural et remettent en question la démocratie représentative locale. Ailleurs, les structures locales engagées dans les dispositifs de la dévolution ne disposent pas plus de reconnaissance juridique, même sous les statuts de coopérative au Mali ou d'association au Niger. Et si d'aucuns ont pu relever des pratiques politiciennes dans la gestion des ressources et du foncier par les élus locaux, et des pratiques de corruption dans les administrations forestières, (Blundo, 2000), il reste que l' échelle "communautaire " et villageoise n'en est pas davantage exempte (Thiaw et Ribot, 2003 ; Bardhan, 2002 ; Boutinot, 2005).

Par ailleurs, La capacité de ces collectifs à produire du consensus serait, dans les approches d'économie institutionnelle, à rechercher dans le capital social que les acteurs détiendraient ou seraient susceptibles de mobiliser. On peut, suivant Ishihara et Unai (2009), questionner la notion de capital social pour expliquer la capacité de ces collectifs à imposer des arrangements entre leurs membres et interroger l'opérationnalité de ce concept. Or cette notion de capital social, bien que recouvrant des acceptions différentes, est utilisée par les bailleurs de fonds, comme par certains des tenants du discours académique, pour expliquer la capacité de mobilisation et de participation des acteurs au sein des comités (Putnam 2007, Coleman 2002, Lin 1982). Or, que le capital social soit un attribut de la structure sociale (qui recouvre confiance et obligations) et facilite l'action collective (Coleman), ou qu'il soit une ressource à mobiliser à titre individuel au sein d'un réseau (Lin), il s'agit d'une hypothèse insuffisante et qui n'est pas vérifiée pour deux raisons. D'une part, parce que, contrairement à ce qu'avancent les théories néo-institutionnelles, le capital social n'est pas simplement une accumulation de stratégies individuelles et de calculs pour créer ou mobiliser des liens sociaux (Ishihara et Unai, 2009) comme s'il s'agissait de seules relations contingentes. D'autre part, parce que, si l'on se réfère à la note de Pierre Bourdieu (1980), et qu'on se place dans un registre critique, on constate que le capital social relève de la production et de l'entretien de liens sociaux plus complexes, issus d'un code partagé et incorporé, c'est-à-dire identifiable dans les pratiques corporelles, les façons de parler, de se vêtir, de se tenir, bref dans un habitus corporel, par les membres de groupes qui se reconnaissent et dont les 
bénéfices qu'ils peuvent en retirer sont d'autant plus grands que ces membres 'valent d'être connus' (puisque déjà connus) (Bourdieu, 1980). Ce capital entretient plus des positions hiérarchiques qu'il ne créé de coordination.

\section{Conclusion}

La socio-anthropologie analyse ici les faits de développement d'un point de vue critique, en regard des présupposés contenus dans les concepts repris aux approches institutionnelles par la Banque mondiale, - tels ceux de coûts de transaction, d'asymétrie d'information, de capital social -afin d'apporter une légitimité académique à ses programmes de bonne gouvernance dans le champ du développement (Olivier de Sardan, 2013 : 288 ; Gautier et al., 2013).

Les contrats de gestion des ressources naturelles apparaissent comme des mécanismes qui ont théoriquement un potentiel de "transparence " et "d'efficience ", selon des approches économiques qui rendent ainsi compte du rapport coût-efficacité de ces dispositifs sans remettre en question les présupposés néo-libéraux qui les sous-tendent (Chomitz et Griffith, 1997). Les politiques publiques de dévolution étudiées préconisent, dans leur procédure de transfert de la gestion des forêts étudiées, des instruments (aménagement forestier participatif, gestion communautaire de base, chartes et codes locaux de gestion) qui ont un double effet : ils engendrent des formes polycentriques de la gouvernance qui contrarient, de ce fait et en même temps, la délégation de pouvoir aux collectivités territoriales. Ce paradoxe se donne à voir dans ses effets concrets dès lors qu'il s'agit d'aborder les dimensions politiques de la figure du contrat. Si les notions de pluralisme institutionnel, de redondance des formes institutionnelles et de polycentrisme auxquelles on prête des vertus démocratiques sont largement traitées dans la littérature du développement en lien avec les approches du " Natural Resource Management » et des Common Pool Resources (Mc Ginnis 1999, Ostrom 1986, Ostrom 2005), on en voit ici les limites. De plus, les notions de transparence, d'empowerment ou de redevabilité qui président, en filigrane, derrière la définition de la bonne gouvernance, n'y paraissent pas adaptées. La dévolution ne peut pas être considérée uniquement comme un projet de gouvernance horizontale car l'usage des conventions locales à l'échelle villageoise sont autant de relais 
des pouvoirs publics pour maintenir le contrôle de ces derniers sur l'accès aux ressources (Ribot et al. 2006). En définitive, des institutions locales ad hoc ainsi multipliées à une échelle infra-citoyenne des collectivités locales élues, vident de leur dimension politique toute notion de représentation. Légitimés par des arguments en termes d'économie de coûts de transaction, peu convaincants à l'épreuve du terrain, les contrats, chartes et codes locaux sont à la fois produits par, et producteurs de relations asymétriques ; ils formalisent, en définitive, de nouveaux modes de gouvernance qui n'ont rien d'horizontal.

\section{Bibliographie}

AGRAWAL, A et GIBSON, C.G., 1999, Enchantment and Disenchantment: The Role of Community in Natural Resource Conservation, World Development, 27(4) : 629-649.

AGRAWAL, A, et RIBOT, J., 1999, Accountability in decentralization: A framework with South Asian and African cases, Journal of Developing Areas, 33 (4) : 473-502.

AGRAWAL, A. et OSTROM, E., 2001, Collective Action Property rights and decentralization in resource use in India and Nepal, Politics and society, 29 : 485-514.

ANDERSON, D. et FISHWICK, R., 1985, Fuelwood Consumption and Deforestation in African Countries: A Review, World Bank Staff Working Paper, $n^{\circ} 704$.

ANDERSSON, K., 2004, Who Talks with Whom? The Role of Repeated Interactions in Decentralized Forest Governance, World Development 32(2): 233-50.

BAKO-ARIFARI, N. et LAURENT JP. eds, 1998, Les dimensions sociales et économiques du développement local et la décentralisation en Afrique au Sud du Sahara. Bulletin de l'APAD, 15.

BARDHAN, P., 2002, Decentralization of Governance and Development, The Journal of Economic Perspectives, vol. 16(4): 185-205.

BENE C. et al., 2009, Power struggle, dispute and alliance over local resources: analyzing 'democratic' decentralization of natural resources through the lens of Africa Inland Fisheries, World Development, 37(12): 1935-1950. 
BERTRAND A. et MONTAGNE, P., 2009, Domestic energy strategies and sustainable management of forest resources in Niger and Mali: management, public property regime, forest taxation and forestry assessment, Bois et Forêts des Tropiques, $\mathrm{n}^{\circ}$ 301: 83-97, http://bft.revuesonline.com.

BIRNER, R et WITTMER, H, 2004, On the 'efficient boundaries of the state': the contribution of transaction-costs economics to the analysis of decentralization and devolution in natural resource management, Environment and Planning C: Government and Policy 22(5): 667-685.

BLAIKIE, P., 2006, Is small really beautiful? Community-based natural resource management in Malawi and Bostwana, World Development, 34(11): 1942-1957.

BLUNDO, G., 2000, Elus locaux et courtiers en développement au Sénégal. Trajectoires politiques, modes de légitimation et stratégies d'alliance, In : Bierschenk T., Chauveau, J.-P., Olivier de Sardan, J.-P., Courtiers en développement. Les villages africains en quête de projets, Paris: APADKarthala : 71-100.

BOURDIEU, P., 1980, Le Capital social. Notes provisoires. Actes de la Recherche en Sciences Sociales, 31 : 2-3.

BOUTINOT, L. et DIOUF C. N., 2006, Quand certaines approches participatives engendrent des formes ambiguës de mobilisation de la société civile. Quelques exemples à propos de la gestion des ressources naturelles au Sénégal, In : Bertrand, A., Karsenty, A.et Montagne, P. (eds), L'Etat et la gestion locale durable des forêts en Afrique francophone et à Madagascar, Paris : L'Harmattan, pp 195-212.

BOUTINOT, L., 2005, La décentralisation de la gestion des ressources forestières au Sénégal : un processus contraint par le marché ?, Bulletin de l'APAD, 26 : 47-67.

BOUTINOT, L., 2004, Etude du PROGEDE dans la gestion décentralisée des ressources forestières au Sénégal, PROGEDE, DEFCCS Dakar, $120 \mathrm{p}$.

BOUTINOT, L, 2002, De la complexité de la décentralisation. Exemple de la gestion des ressources forestières au Sénégal. Bulletin de l'APAD, 23 [En ligne], mis en ligne le 15 décembre 2005, URL : http://apad.revues.org/5

BROMLEY, D.W., 1991, Environment and Economy: Property Rights and Public Policy, Oxford: Blackwell Publishers, 247p.

BROUSSEAU, E et GLACHANT, J.M., 2002, The Economics of Contracts. Theories and Application, Cambridge : Cambridge University Press, 579 p. 
COLEMAN, E.A. et FLEISCHMAN, F.D., 2011, Comparing forest decentralization in Bolivia, Kenya, Mexico, Uuganda, World Development, 40(4): 836-849.

COLEMAN, J. S., 1988, Social capital in the creation of human capital, American Journal of sociology, 94 : S95-S120.

CROOK, R. et MANOR, J., 1998, Democracy and decentralization in South Asia and West Africa, Cambridge : Cambridge University Press.

DJIRE M., 2004, Les conventions locales au Mali : une grande nébuleuse juridique et un pragmatisme en Gestion des Ressources Naturelles, IIED «Réussir la décentralisation $", n^{\circ} 24$.

ECKHOLM, E.R., FOLLEY, G., BARNARD, G. et TIMBERLAKE, L., 1984, Firewood: the energy crisis that won't go away, London UK, Earthscan.

FAYE, P., 2006, Décentralisation, pluralisme institutionnel et démocratie locale. Etude de cas de la gestion des massifs forestiers de Missirah/Kothiary, Région de Tambacounda, Sénégal, CODESRIA - Monograph's Series, Dakar.

GAUTIER, D., HAUDIDIER, D. HAYA, I. et GAZULL L., 2008, Le marché rural de bois au Mali à l'épreuve du temps: une innovation en friche, In :Méral P. et Froger, G., La gestion concertée des ressources naturelles : l'épreuve du temps, Paris, Karthala, pp 67-84.

GAUTIER, D., BENJAMINSEN, T., GAZULL, L., ANTONA, M., 2013, Neoliberal Forest Reform in Mali: Adverse Effects of a World Bank "Success", Society \& Natural Resources: An International Journal, Volume 26, Issue 6, p. 702716 DOI:10.1080/08941920.2012.709587.

GIBSON, C. et LEHOUCQ, F., 2003, The local politics of decentralized environmental policy in Guatemala, Journal of Environment and Development, 12 (1): 28-49.

GRANIER, L., 2006, Les conventions locales de gestion des ressources naturelles et de l'environnement. Légalité et cohérence en droit sénégalais, UICN, Gland, Suisse et Cambridge, Royaume-Uni, $44 \mathrm{p}$.

HAUBERT, M., 2000, "L'idéologie de la société civile ", In : Haubert M. et Rey P.P. (coord.), Les sociétés civiles face au marché. Le changement social dans le monde postcolonial, Paris, Karthala, pp. 13 -86.

HAUTDIDIER, B. et GAUTIER, D., 2005, What local benefits does the implementation of rural wood markets in Mali generate? In M. A. F. Ros-Thonen and A. J. Dietz (eds) African forests between nature and livelihood resources: 
Interdisciplinary studies in conservation and forest management, Ceredigion, UK: Edwin Mellen Press, pp. 191-220.

HAUTDIDIER, B., BOUTINOT, L. et GAUTIER, D., 2004, La mise en place d marchés ruraux de bois au Mali : un événement social et territorial, L'Espace géographie, 33, $n^{\circ} 4$, pp. 289- 305.

HUFTY, M., 2007, La gouvernance est-elle un concept opérationnel ?, Fédéralisme et régionalisme, vol. $7 \mathrm{n}^{\circ} 2$ - Société civile, globalisation, gouvernance : aux origines d'un nouvel ordre politique ?http://popups.ulg.ac.be/federalisme/document.php?id=635.

ISHIHARA, H. et UNAI, P, 2009, Social capital in community level environmental governance: A critique, Ecological Economic, 68 (2009) : 1549-1562.

KASSIBO, B., 1997, La décentralisation au Mali: état des lieux, Bulletin de l'APAD, $\mathrm{n}^{\circ} 14: 1-20$.

KASSIBO, B., 2010, Local government and forest resource governance in Mali: Taxation and decentralized management of forest resources in Siby Rural Commune, In L. A. German, A. Karsenty, and A.-M. Tiani (eds) GoverningAfrica's forests in a globalized world, ed, London, UK: Earthscan: 191-206.

LARSON, A M., 2005, Democratic decentralization in the forestry sector: lessons learned from Africa, Asia and Latina America, In: Colfer, C., Capistrano D., The politics of decentralization: forests, power and people, Earthscan Forestry Library, pp 32- 63.

LAVIGNE DELVILLE, P., 2006, Conditions pour une gestion décentralisée des ressources naturelles. Entre " community failures ", " market failures » et "state failures", construire de nouveaux communs, In : BERTRAND, A., KARSENTY, A. et MONTAGNE, P. (eds.), L'Etat et la gestion locale durable des forêts en Afrique francophone et à Madagascar, Paris, L'Harmattan, pp. 153-173.

LAVIGNE DELVILLE, P., HOCHET, P., 2005,Construire une gestion négociée et durable des ressources naturelles renouvelables en Afrique de l'Ouest, Rapport final de l'étude AFD/UE , Paris, GRET, $180 \mathrm{p}$.

LIN, N., 1982, Social capital, Cambridge, Cambridge University Press, 281 p.

Mc GINNIS, M., 1999, Introduction in Polycentric Governance and Development. Readings from the Workshop in Political Theory and Policy Analysis Ann Harbor: University of Michigan Press, On line http://www.indiana.edu/ workshop/publications/materials/volume1.html. 
MEINZEN DICK, R., KNOX, A., PLACE, F., and SWALLOW, B.M. (eds.), 2002, Innovation in natural resource management: The role of property rights and collective action in developing countries. Baltimore, MD: Johns Hopkins University Press and International Food Policy Research Institute. http://www.ifpri.org/pubs/jhu/innovnrm.htm

MERMET, L., BILLE, R., LEROY, M., NARCY, J.B. et POUX, X., 2006, L'analyse stratégique de la gestion environnementale : un cadre théorique pour penser l'efficacité en matière d'environnement, Natures Sciences Sociétés, 13(2005) : 127-137.

NOPPEN, D., KERKHOF, P., et HESSE, C., 2004, Les marchés ruraux de bois énergie au Niger. Bilan de l'appui à la Stratégie Energie Domestique du Niger, 1989-2003, Rapport IIED/Danida [on line : http://www.eartprint.com].

NORTH, D.C., 1990, Institutions, Institutional change and Economic Performance, Cambridge: Cambridge University Press.

OLIVIER DE SARDAN, J-P., 2013, Embeddedness and informal norms: institutionalism and anthropology, Critique of Anthropology, 33(3) : 280299.

OLIVIER DE SARDAN, J-P., 2010, Gouvernance locale et biens publics au Niger, Working Paper $\mathrm{n}^{\circ} 1$, Juillet, ODI, Londres, 34 pages.

OSTROM, E., 1986, An Agenda for the Study of Institutions, Public Choice, 48 : 3-25.

OSTROM, E., 2005, Understanding Institutional Diversity, Princeton NJ : Princeton University Press.

OSTROM, E., 2007, A diagnosis approach for going beyond panaceas. Proceedings of the National Academy of sciences USA, 104: 15181-15187.

POLANYI, K., 1983 [1 e $^{\mathrm{e}}$ ed. angl. 1944], La Grande Transformation. Aux origines politiques et économiques de notre temps, Paris, Gallimard, $420 \mathrm{p}$.

PUTNAM, R., 2007, E Pluribus Unum, diversity and community in the twenty first century, Scandinavian Political Studies, 30(2), 137-174

RÉPUBLIQUE DU SÉNÉGAL, 1987, Arrêté portant organisation de la compagne d'exploitation forestière pour l'année 1986-1987, n 000054/ MPN/ DEF/ DPF, Dakar.

RÉPUBLIQUE DU SÉNÉGAL, 2010, Arrêté ministériel, N³276 MEPNBRLA-DEFCCS en date du 8 avril 2010, portant organisation de la campagne d'exploitation forestière, J.O. $N^{\circ} 6539$ du samedi 7 août 2010. 
RIBOT, J., AGRAWAL, A., et LARSON, A., 2006, Recentralizing while decentralizing : how national governements reappropriate forest resources, World Development, 34(11), 1864-1886.

RIBOT, J.C., 1990, Politique forestière et filière du charbon de bois, In : Bergeret, A., L'arbre nourricier en pays sahélien, Paris, Editions de la Maison des Sciences de l'Homme : 139-183.

RIBOT, J.C., 2002, Democratic decentralization of natural resources: institutionalizing popular participation, Washington D.C., World Resource Institute, 30p.

RIBOT, J.C., 2004, Waiting for democracy. The Politics of Choice of Natural Resource Decentralisation, Washington DC, World Resource Institute report, 189p.

THIAW, S., et RIBOT, J.-C., 2003, Privileging Outsiders, Excluding Insiders. Forest access through Village Chief in Senegal, Communication for The International Conference on Competing Jurisdictions: Settling land Claims in Africa, Vrije Universiteit Amsterdam, 24-27 sept 2003.

THOMSON, J.T., 2000, Special districts: an institutional tool for improved common pool resource management, $8^{\mathrm{e}}$ Conférence IASCP, Bloomignton, Indiana.

YAMBA, B., 2001, Les politiques publiques de gestion des ressources ligneuses au Niger, In : Idrissa K. (dir), Le Niger: Etat et Démocratie, Paris, I'Harmattan : $127-172$.

\section{Aiviviv}

Martine Antona est responsable de l UR GREEN au Cirad

Laurence Boutinot est socio-anhropologue au Cirad

mail : Martine.antona@cirad.fr

mail : Laurence.boutinot@cirad.fr

Brehima Kassibo $* * * * *$ 
